\title{
Classification of Immature and Mature Coffee Beans Using RGB Values and Machine Learning Algorithms
}

\author{
William R. Eustaquio ${ }^{1}$, Jesusimo L. Dioses Jr. ${ }^{2}$ \\ ${ }^{1}$ College of Arts and Sciences, , Isabela State University, Echague, Isabela, Philippines, \\ william.r.eustaquio@isu.edu.ph \\ ${ }^{2}$ College of Computing Science, Information and Communication Technology, Isabela State University, \\ Echague, Isabela , Philippines, jesusimo.l.dioses@gmail.com
}

\begin{abstract}
The practice of coffee farmers to harvest all coffee cherries when $75 \%$ of it are full red in a coffee tree gave rise to immature coffee beans. Immature coffee beans are green and yellow coffee cherries which were harvested earlier before they turn fully red, in order to reduce the operating cost of the coffee farmers. This practice greatly lowers the grade of coffee. Visually immature and mature coffee beans are different. Immature coffee beans are darker in color. When they are mixed, it will be very difficult to distinguish one from the other by visual inspection. This paper is about using image processing to extract RGB color features of the mature and immature coffee beans and used the extracted RGB values for classification using the 23 machine learning classifiers of Matlab's Classification Learner App. The best in terms of accuracy and speed in training is the Quadratic Support Vector Machine which has a value of 94 $\%$ and 0.62 seconds respectively.
\end{abstract}

Key words : Immature coffee beans; Mature coffee beans; MATLAB Classification Learner App; RGB values.

\section{INTRODUCTION}

Coffee is one of the world's most important food commodities[1]. Among all world commodities it is second to crude oil in importance[2]. Coffee is being produced and the main agricultural export product of almost sixty tropical and sub-tropical countries[3]. Quality of coffee beans are evaluated using many criteria among which are size, color, shape, roast potential, flavor or cup quality and the presence of defects[4]. The presence of defects affect the quality of coffee and is associated with harvesting and pre-processing procedures[5]. The different types of coffee bean defects have been discussed in the Department of Agriculture of the Philippines in the Philippine National Standards for Green

Coffee Bean Specifications. According to this standards, there are nine types of defects namely black beans, infested beans, broken beans, immature beans, husk fragments, fermented or sour beans, admixture and foreign matter [6].Based on color, if the coffee bean is greenish, grayish or spongy white, the coffee bean is immature. Immature coffee beans is a result of harvesting coffee fruit even though they are still green or yellow color instead of full ripe red color. One batch harvesting were done because it saves time wherein all coffee cherries are harvested at one time when $75 \%$ were red, instead of waiting for green and yellow fruit to turn full red[7]. Green, yellow and red coffee fruits when dried are almost impossible to differentiate visually but the major drawback is the quality or grade of a batch of coffee beans are greatly downgraded because of the presence of immature beans[8].

The common procedure to separate the defective coffee beans is by manual inspection and picking and separating the defective beans. This method is time consuming and is subjective, because it is dependent on the conditions of the person performing the defect selection[9]. Manual inspection systems is based on human judgement and may vary from one person to another, even though both persons are regarded as experts[10]. The objective of this paper is to automate the classification of mature and immature coffee beans using image processing and machine learning algorithms. Image processing is an effective instrument in extracting features of an object [11-30] and machine learning algorithms are efficient tools in discriminating object using the extracted features[9]. The combine image processing and machine learning approach of classification has been tested and found effective in terms of high accuracy in a number of studies [31-32].

\section{METHODOLOGY}

Coffee beans from harvest season of January to March 2019 were used in this study. There are two coffee bean groups used, the first group are the mature coffee beans which are the fully red coffee cherries picked from the Robusta coffee tree. The second group are the immature coffee beans composed of green and yellow coffee cherries picked from the same coffee tree. Both groups were dried 
under the sun for the same duration in different containers. After drying the husk of the coffee beans were removed and stored in a cool dry place. After a year of storage, the taking of images and feature extraction was done on March 2020. Figure 1 shows the coffee bean samples used in this study which are composed of green, yellow and red beans.

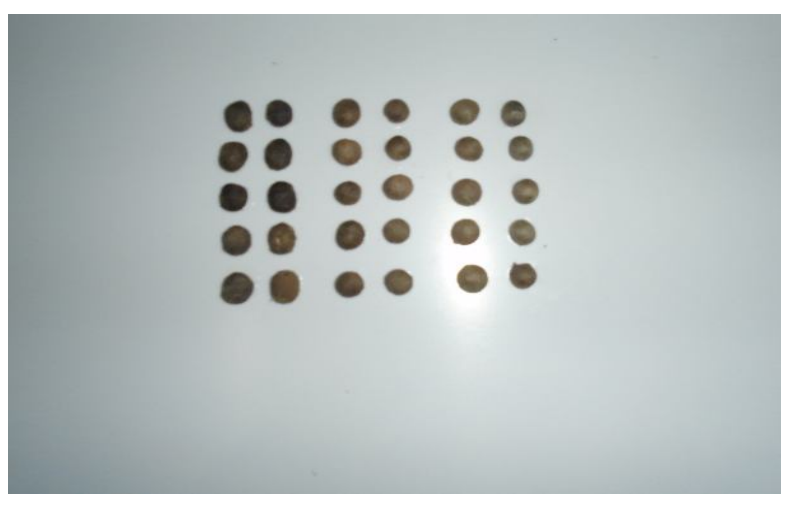

Figure 1: Immature and Mature Coffee Beans

In Figure 1, the first four columns are dried immature beans and the last two columns are mature beans.

\section{B. Feature Extraction}

It was observed that the mature and immature coffee beans differ in terms of color visually. The researcher extracted the RGB features of the two bean groups to prove whether they can be differentiated using image processing and machine learning classifiers. A total of 100 mature beans and 100 immature beans were used in the study. The coffee beans were placed in panel board with white background and the images were taken by a A4tech PK $-835 \mathrm{G}$ webcam, situated directly above the coffee beans at a height of $13.5 \mathrm{cms}$. For lighting, a two pieces of 3 inch LED strips were put at the two sides of the camera at a distance of 2 inches. The images were saved in a computer and the RGB features were extracted using a python image processing program as shown in Figure 2.



Figure 2: Python program for extracting R,G,B values.

\section{Classification}

The classifier used for differentiating mature from immature coffee beans are the 23 machine learning algorithms of Matlab's Classification Learner App (CLA). The extracted RGB color features were inputted to the CLA using 5 folds, this means that 80 beans were used for training and the 20 beans were used for testing and validation. The accuracy and training time were taken into account to determine which is the best classifier of the mature and immature coffee beans.

\section{RESULTS AND DISCUSSION}

The extracted features of mature and immature coffee beans is shown in Table 1. It can be seen in Table 1 that the RGB values for mature and immature coffee beans are overlapping. It can also be observe that RGB values of mature coffee beans are lower than the RGB values of immature coffee beans.

Table 1: RGB Range of Values for Mature and Immature Coffee Beans

\begin{tabular}{|c|c|c|c|}
\hline $\begin{array}{c}\text { Coffee } \\
\text { Bean Type }\end{array}$ & Red & Green & Blue \\
\hline $\begin{array}{c}\text { Mature } \\
\text { Coffee } \\
\text { Beans }\end{array}$ & 74 to 143 & 89 to 163 & 89 to 159 \\
\hline $\begin{array}{c}\text { Immature } \\
\text { Coffee } \\
\text { Beans }\end{array}$ & 90 to 150 & 100 to 168 & 104 to 164 \\
\hline
\end{tabular}

Table 2 shows the classification results for the 23 machine learning algorithms

Table 2: Classification Results

\begin{tabular}{|c|c|c|}
\hline Classifier Type & $\begin{array}{c}\text { Accuracy } \\
(\mathbf{\%})\end{array}$ & $\begin{array}{c}\text { Training Time } \\
\text { (Seconds) }\end{array}$ \\
\hline Fine Tree & 84 & 27.91 \\
\hline Medium Tree & 84 & 0.32 \\
\hline Coarse Tree & 81.5 & 0.21 \\
\hline $\begin{array}{c}\text { Linear } \\
\text { Discriminant }\end{array}$ & 94 & 7.12 \\
\hline $\begin{array}{c}\text { Quadratic } \\
\text { Discriminant }\end{array}$ & 93 & 0.97 \\
\hline Logistic Regression & 93 & 26.30 \\
\hline $\begin{array}{c}\text { Linear Support } \\
\text { Vector Machine } \\
\text { (SVM) }\end{array}$ & 94 & 6.33 \\
\hline Quadratic SVM & 94 & 0.62 \\
\hline
\end{tabular}


William R. Eustaquio et al., International Journal of Emerging Trends in Engineering Research, 8(7), July 2020, 3016 - 3022

\begin{tabular}{|c|c|c|}
\hline Cubic SVM & 93 & 11.4 \\
\hline $\begin{array}{c}\text { Fine Gaussian } \\
\text { SVM }\end{array}$ & 93 & 0.35 \\
\hline $\begin{array}{c}\text { Medium Gaussian } \\
\text { SVM }\end{array}$ & 92.5 & 0.21 \\
\hline $\begin{array}{c}\text { Coarse Gaussian } \\
\text { SVM }\end{array}$ & 80.5 & 0.19 \\
\hline Fine KNN & 84 & 3.26 \\
\hline Medium KNN & 89 & 0.21 \\
\hline Coarse KNN & 74.5 & 0.20 \\
\hline Cosine KNN & 92 & 0.53 \\
\hline Cubic KNN & 91.5 & 0.25 \\
\hline Weighted KNN & 87.5 & 0.29 \\
\hline Boosted Trees & 82.5 & 5.67 \\
\hline Bagged Trees & 85 & 3.05 \\
\hline $\begin{array}{c}\text { Subspace } \\
\text { Discriminant }\end{array}$ & 93 & 2.59 \\
\hline Subspace KNN & 81.5 & 2.46 \\
\hline RUS Boosted Trees & 84.5 & 5.25 \\
\hline
\end{tabular}

Table 2 also shows that the Linear SVM and Quadratic SVM both achieved the highest accuracy of $94 \%$, however in terms of training time the fastest was achieved by quadratic SVM with 0.64 seconds, compared to 6.33 seconds of linear SVM. The CLA setting known as the Principal Component Analysis (PCA) was not used in the classification.

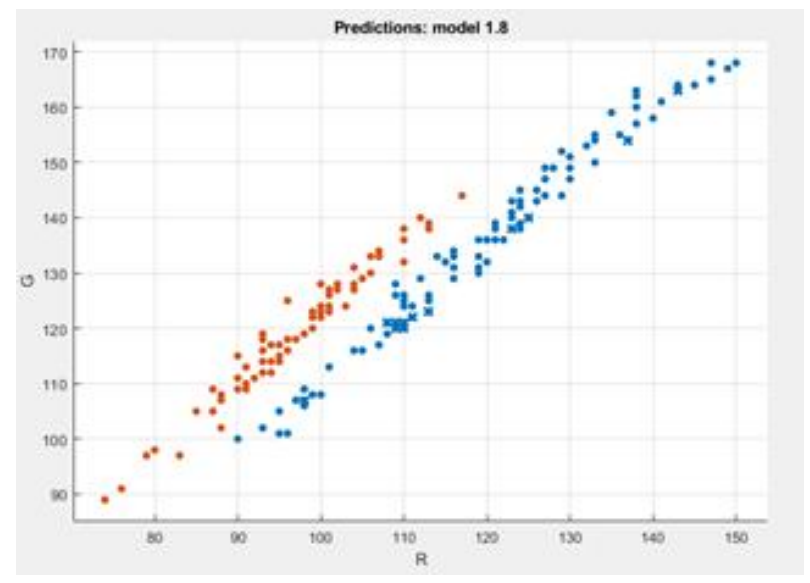

Figure 3: Scatter Plot of the Quadratic SVM Classifier

Figure 3 shows that the 10 features are overlapping for civet coffee and normal robusta coffee beans.

Figure 4 shows the Confusion Matrix of the Quadratic SVM classifier.

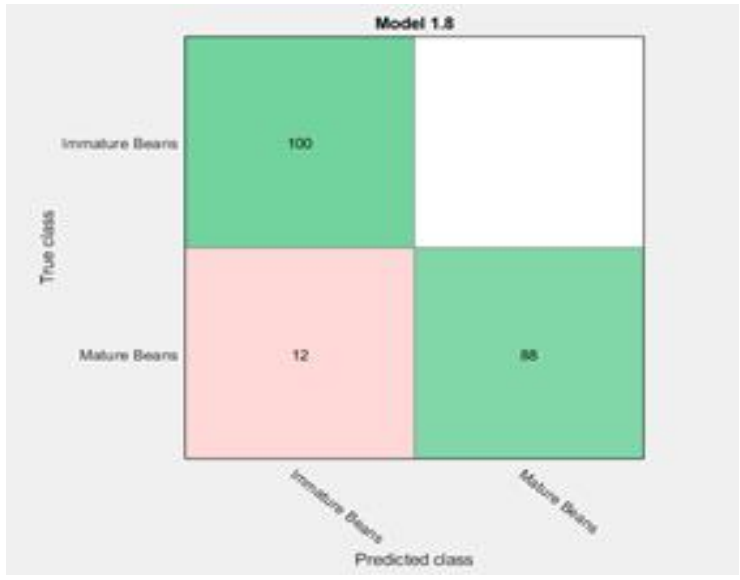

Figure 4: Confusion Matrix of the Quadratic SVM Classifier

It can be seen in Figure 4, the confusion matrix that the Quadratic SVM Classifier was able to correctly identify immature coffee beans by $100 \%$ and mature coffee beans by only $88 \%$.

Figure 5 shows the Receiver Operating Characteristic (ROC) curve of the Quadratic SVM.

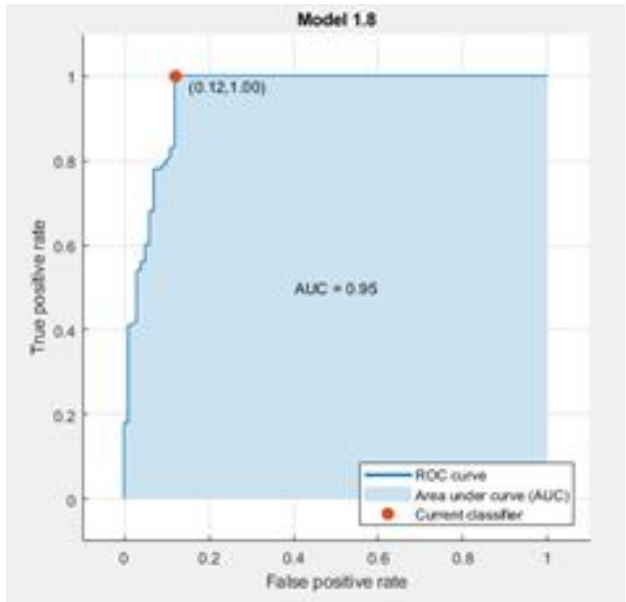

Figure 5 : The ROC Curve of Quadratic SVM Classifier

In Figure 5 the area under the curve (AUC) of the Quadratic SVM is equal to 0.95 . The more the AUC value approaches 1, the higher the accuracy of the classifier. The AUC value of 0.95 of Quadratic SVM is the best AUC value compared to other classifiers.

Figure 6 show the parallel coordinates plot of the quadratic SVM . 


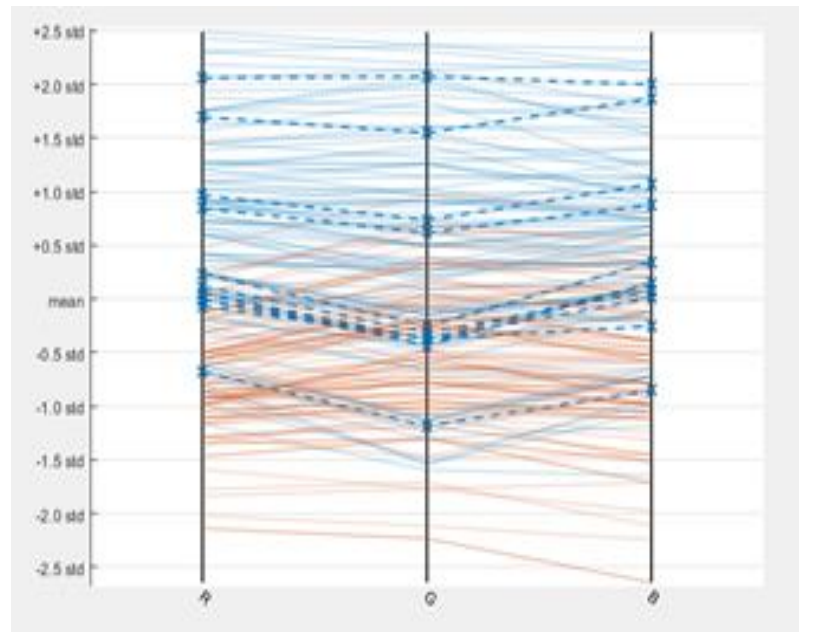

Figure 6: The Parallel Coordinates Plot of Quadratic SVM Classifier

The parallel coordinates plot shows the useful variables or features namely the RGB values of the mature and immature coffee beans that contributed to their discrimination. It can be seen in the plot that the RGB features of the mature and immature coffee beans overlapped by still the Quadratic SVM algorithm was able to discriminate the two bean group with $94 \%$ accuracy.

\section{CONCLUSION}

The paper is about answering the research question whether mature and immature coffee beans can be differentiated using RGB features extracted using image processing and classified using machine learning algorithms. It can be concluded that the RGB of mature beans differ with RGB of immature beans. This was achieved by using the Quadratic SVM algorithm with $94 \%$ accuracy at a training speed of 0.62 seconds.

\section{REFERENCES}

[1] K. P. P. Nair, "6-Coffee," in The Agronomy and Economy of Important Tree Crops of the Developing World, Elsevier, 2010, pp. 181-208. https://doi.org/10.1016/B978-0-12-384677-8.000060

[2] P. Esquivel and V. M. Jiménez, "Functional properties of coffee and coffee by-products," Food Res. Int., vol. 46, no. 2, pp. 488-495, 2012.

[3] E. K. Kemsley, S. Ruault, and R. H. Wilson, "Discrimination between Coffe arabica and Coffea canephora variant robusta beans using infrared spectroscopy," Food Chem., vol. 8146, no. 95, pp. 321-326, 1995.

https://doi.org/10.1016/0308-8146(95)00030-M

[4] A. Datov and Y.-C. Lin, "Classification and Grading of Green Coffee Beans in Asia," 2019.

[5] A. P. Craig, A. S. Franca, and L. S. Oliveira,
"Discrimination between Immature and Mature Green Coffees by Attenuated Total Reflectance and Diffuse Reflectance Fourier Transform Infrared Spectroscopy," J. Food Sci., vol. 76, no. 8, 2011.

[6] Philippine National Standards PNS/BAFPS 01:2012, "PHILIPPINE NATIONAL Green Coffee Beans Specifications," 2012. [Online]. Available: http://www.bafps.da.gov.ph/index.php/standard-for mulation/philippine-national-standards?start $=20$.

[7] R. G. Idago and R. S. Dela Cruz, Value Chain Improvement of Robusta, vol. 5, no. 1. 2012.

[8] M. N. Clifford and T. Kazi, "The Influence of Coffee Bean Maturity on the Content of Chlorogenic Acids, Caffeine and Trigonelline," Food Chem., vol. 26, pp. 59-69, 1987.

https://doi.org/10.1016/0308-8146(87)90167-1

[9] E. R. Arboleda, "Comparing Performances of Data Mining Algorithms for Classification of Green Coffee Beans," Int. J. Eng. Adv. Technol., vol. 8, no. 5, pp. 1563-1567, 2019.

[10] E. R. Arboleda, A. C. Fajardo, and R. P. Medina, "Classification of Coffee Bean Species Using Image Processing, Artificial Neural Network and K Nearest Neighbors," in 2018 IEEE International Conference on Innovative Research and Development (ICIRD), 2018, no. May, pp. 1-5.

[11] E. R. Arboleda, A. C. Fajardo, and R. P. Medina, "Green coffee beans feature extractor using image processing," TELKOMNIKA (Telecommunication Comput. Electron. Control., vol. 18, no. 4, p. 2027, 2020.

[12] A. S. Alon and J. L. Dioses, "A machine vision detection of unauthorized on-street roadside parking in restricted zone: An experimental simulated barangay-environment," Int. J. Emerg. Trends Eng. Res., vol. 8, no. 4, pp. 1056-1061, 2020. https://doi.org/10.30534/ijeter/2020/17842020

[13] J. A. A. Garcia, E. R. Arboleda, and E. M. Galas, "Identification Of Visually Similar Vegetable Seeds Using Image Processing And Fuzzy Logic," Int. J. Sci. Technol. Res., vol. 9, no. 02, pp. 4925-4928, 2020.

[14] A. J. G. Gonzales, J. A. D. Sosa, E. R. Arboleda, and E. M. Galas, "Filtering Of Faded Coffee Beans Using Image Processing," Int. J. Sci. Technol. Res., vol. 8, no. 12, pp. 3634-3637, 2019.

[15] F. B. Vidad III, E. R. Arboleda, J. L. Dioses Jr, and R. M. Dellosa, "Morphological Based Seedling Comparison Of Natural And Hybrid Lemon," Int. J. Sci. Technol. Res., vol. 9, no. 02, 2020.

[16] E. J. Olaes, E. R. Arboleda, J. L. D. Jr, and R. M. Dellosa, "Bell Pepper And Chili Pepper Classification : An Application Of Image Processing And Fuzzy Logic," Int. J. Sci. Technol. Res., vol. 9, no. 02, pp. 4833-4839, 2020.

[17] C. L. Macalalad, E. R. Arboleda, A. A. Andilab, and R. M. Dellosa, "Morphological Based Grain 
William R. Eustaquio et al., International Journal of Emerging Trends in Engineering Research, 8(7), July 2020, 3016 - 3022

Comparison of Three Rice Grain Variety," Int. Jounal Sci. Technol. Res., vol. 8, no. 08, pp. 1446-1450, 2019.

[18] R. A. Magbayao, E. R. Arboleda, and E. M. Galas, "Identification Of Asian Green Mussel Perna Viridis ' Sex Using Image Processing, Fuzzy Logic And K Nearest Neighbor," Int. J. Sci. Technol. Res., vol. 9, no. $01,2020$.

[19] J. N. C. Sarino, M. M. Bayas, E. R. Arboleda, E. C. Guevarra, and R. M. Dellosa, "Classification Of Coffee Bean Degree Of Roast Using Image Processing And Neural Network," Int. J. Sci. Technol. Res., vol. 8, no. 10, pp. 3231-3233, 2019.

[20] V. M. D. Manalo, E. R. Arboleda, J. L. D. Jr, and R. M. Dellosa, "Differentiation Among Lettuce ( L . Sativa ) Seed Varieties Grown In Gourmet Farms , Silang Cavite, Philippines Using Image Processing With Fuzzy Logic And Knn As Classifiers," Int. J. Sci. Technol. Res., vol. 8, no. 10, pp. 8-11, 2019.

[21] M. J. L. Jacoba and E. R. Arboleda, "Identification of rain cloud amount, rain intensity and possible chance of flooding using image processing and fuzzy logic," ARPN J. Eng. Appl. Sci., vol. 14, no. 14, pp. 2563-2568, 2019.

[22] J. G. C. Rancapan, E. R. Arboleda, J. L. D. Jr, and R. M. Dellosa, "Egg Fertility Detection Using Image Processing And Fuzzy Logic," Int. J. Sci. Technol. Res., vol. 8, no. 10, pp. 3228-3230, 2019.

[23] A. S. Alon and J. L. Dioses, "Machine vision recognition system for iceberg lettuce health condition on raspberry pi 4b: A mobile net ssd v2 inference approach," Int. J. Emerg. Trends Eng. Res., vol. 8, no. 4, pp. 1073-1078, 2020.

https://doi.org/10.30534/ijeter/2020/20842020

[24] A. E. Andaya, E. R. Arboleda, A. A. Andilab, and R. M. Dellosa, "Meat Marbling Scoring Using Image Processing with Fuzzy Logic Based Classifier," Int. J. Sci. Technol. Res., vol. 8, no. 08, pp. 1442-1445, 2019.

[25] R. E. T. Bae, E. R. Arboleda, A. Andilab, and R. M. Dellosa, "Implementation Of Template Matching, Fuzzy Logic And K Nearest Neighbor Classifier On Philippine Banknote Recognition System," Int. J. Sci. Technol. Res., vol. 8, no. 08, pp. 1451-1453, 2019.

[26] D. L. Bersabal, E. R. Arboleda, and E. M. Galas, "Coffee Bean Recognition Using Shape Features Using Decision Trees And Ensemble Classifiers," Int. J. Sci. Technol. Res., vol. 9, no. 02, 2020.

[27] V. A. C. Austria, E. R. Arboleda, and E. M. Galas, "Image Processing Of Clean And Dirty Dishes To Design And Construct A Fuzzy Logic Dishwasher," Int. J. Sci. Technol. Res., vol. 8, no. 12, pp. 8-11, 2019.

[28] A. M. Castillo, R. D. Aradanas, E. R. Arboleda, A. A. Dizon, and R. M. Dellosa, "Coffee Type Classification Using Gray Level Co- Occurrence Matrix Feature Extraction And The Artificial Neural
Network Classifier," Int. J. Sci. Technol. Res., vol. 8, no. 10, pp. 1463-1465, 2019.

[29] N. O. Delgado, E. R. Arboleda, J. L. Dioses Jr, and R. M. Dellosa, "Identification Of Mango Leaves Using Artificial Intelligence," Int. J. Sci. Technol. Res., vol. 8, no. 12, pp. 2864-2868, 2019.

[30] "Fuzzy Logic Based Vehicular Congestion Estimation Monitoring System Using Image Processing and KNN Classifier," Int. J. Sci. Technol. Res., vol. 8, no. 08, 2019.

[31] J. L. Dioses, "Classification of pepper seeds using data mining algorithms," Int. J. Adv. Trends Comput. Sci. Eng., vol. 9, no. 2, pp. 1460-1464, 2020. https://doi.org/10.30534/ijatcse/2020/84922020

[32] J. L. Dioses, "Discrimination of civet coffee using image processing and machine learning," Int. J. Emerg. Trends Eng. Res., vol. 8, no. 4, pp. 1067-1072, 2020.

https://doi.org/10.30534/ijeter/2020/19842020

\section{APPENDIX}

The Dataset Of The Study

\begin{tabular}{|c|c|c|c|c|}
\hline Sample & $\mathrm{R}$ & $\mathrm{G}$ & $\mathrm{B}$ & Classes \\
\hline 1 & 88 & 108 & 109 & Mature Beans \\
\hline 2 & 95 & 115 & 118 & Mature Beans \\
\hline 3 & 97 & 118 & 121 & Mature Beans \\
\hline 4 & 95 & 115 & 117 & Mature Beans \\
\hline 5 & 103 & 124 & 127 & Mature Beans \\
\hline 6 & 99 & 120 & 122 & Mature Beans \\
\hline 7 & 110 & 132 & 134 & Mature Beans \\
\hline 8 & 104 & 128 & 127 & Mature Beans \\
\hline 9 & 88 & 102 & 107 & Mature Beans \\
\hline 10 & 92 & 111 & 113 & Mature Beans \\
\hline 11 & 95 & 114 & 116 & Mature Beans \\
\hline 12 & 91 & 109 & 112 & Mature Beans \\
\hline 13 & 90 & 111 & 112 & Mature Beans \\
\hline 14 & 83 & 97 & 103 & Mature Beans \\
\hline 15 & 76 & 91 & 95 & Mature Beans \\
\hline 16 & 91 & 113 & 117 & Mature Beans \\
\hline 17 & 93 & 118 & 120 & Mature Beans \\
\hline 18 & 102 & 127 & 131 & Mature Beans \\
\hline 19 & 107 & 133 & 132 & Mature Beans \\
\hline 20 & 100 & 128 & 127 & Mature Beans \\
\hline 21 & 107 & 134 & 133 & Mature Beans \\
\hline 22 & 101 & 126 & 129 & Mature Beans \\
\hline 23 & 96 & 125 & 123 & Mature Beans \\
\hline 24 & 106 & 133 & 132 & Mature Beans \\
\hline 25 & 100 & 124 & 126 & Mature Beans \\
\hline
\end{tabular}


William R. Eustaquio et al., International Journal of Emerging Trends in Engineering Research, 8(7), July 2020, 3016 - 3022

\begin{tabular}{|c|c|c|c|c|}
\hline 26 & 102 & 128 & 130 & Mature Beans \\
\hline 27 & 90 & 115 & 114 & Mature Beans \\
\hline 28 & 93 & 116 & 119 & Mature Beans \\
\hline 29 & 93 & 119 & 117 & Mature Beans \\
\hline 30 & 107 & 133 & 134 & Mature Beans \\
\hline 31 & 101 & 124 & 126 & Mature Beans \\
\hline 32 & 107 & 134 & 129 & Mature Beans \\
\hline 33 & 106 & 130 & 129 & Mature Beans \\
\hline 34 & 104 & 131 & 126 & Mature Beans \\
\hline 35 & 106 & 130 & 130 & Mature Beans \\
\hline 36 & 98 & 119 & 120 & Mature Beans \\
\hline 37 & 104 & 127 & 128 & Mature Beans \\
\hline 38 & 95 & 117 & 117 & Mature Beans \\
\hline 39 & 101 & 127 & 123 & Mature Beans \\
\hline 40 & 93 & 114 & 116 & Mature Beans \\
\hline 41 & 99 & 123 & 121 & Mature Beans \\
\hline 42 & 80 & 98 & 97 & Mature Beans \\
\hline 43 & 93 & 119 & 114 & Mature Beans \\
\hline 44 & 79 & 97 & 99 & Mature Beans \\
\hline 45 & 110 & 136 & 135 & Mature Beans \\
\hline 46 & 105 & 129 & 131 & Mature Beans \\
\hline 47 & 96 & 116 & 121 & Mature Beans \\
\hline 48 & 99 & 123 & 123 & Mature Beans \\
\hline 49 & 117 & 144 & 141 & Mature Beans \\
\hline 50 & 113 & 139 & 137 & Mature Beans \\
\hline 51 & 112 & 140 & 136 & Mature Beans \\
\hline 52 & 113 & 138 & 138 & Mature Beans \\
\hline 53 & 100 & 122 & 121 & Mature Beans \\
\hline 54 & 100 & 123 & 123 & Mature Beans \\
\hline 55 & 110 & 138 & 134 & Mature Beans \\
\hline 56 & 93 & 112 & 116 & Mature Beans \\
\hline 57 & 99 & 122 & 121 & Mature Beans \\
\hline 58 & 91 & 110 & 113 & Mature Beans \\
\hline 59 & 90 & 109 & 110 & Mature Beans \\
\hline 60 & 101 & 126 & 123 & Mature Beans \\
\hline 61 & 100 & 124 & 120 & Mature Beans \\
\hline 62 & 94 & 117 & 117 & Mature Beans \\
\hline 63 & 88 & 107 & 107 & Mature Beans \\
\hline 64 & 101 & 123 & 122 & Mature Beans \\
\hline 65 & 94 & 112 & 114 & Mature Beans \\
\hline 66 & 92 & 111 & 113 & Mature Beans \\
\hline 67 & 94 & 114 & 114 & Mature Beans \\
\hline 68 & 96 & 118 & 115 & Mature Beans \\
\hline 69 & 91 & 110 & 112 & Mature Beans \\
\hline
\end{tabular}

\begin{tabular}{|c|c|c|c|c|}
\hline 70 & 87 & 105 & 106 & Mature Beans \\
\hline 71 & 94 & 114 & 114 & Mature Beans \\
\hline 72 & 85 & 105 & 103 & Mature Beans \\
\hline 73 & 74 & 89 & 89 & Mature Beans \\
\hline 74 & 87 & 109 & 106 & Mature Beans \\
\hline 75 & 101 & 126 & 123 & Mature Beans \\
\hline 76 & 100 & 124 & 120 & Mature Beans \\
\hline 77 & 94 & 117 & 117 & Mature Beans \\
\hline 78 & 88 & 107 & 107 & Mature Beans \\
\hline 79 & 101 & 123 & 122 & Mature Beans \\
\hline 80 & 94 & 112 & 114 & Mature Beans \\
\hline 81 & 92 & 111 & 113 & Mature Beans \\
\hline 82 & 94 & 114 & 114 & Mature Beans \\
\hline 83 & 96 & 118 & 115 & Mature Beans \\
\hline 84 & 91 & 110 & 112 & Mature Beans \\
\hline 85 & 87 & 105 & 106 & Mature Beans \\
\hline 86 & 94 & 114 & 114 & Mature Beans \\
\hline 87 & 85 & 105 & 103 & Mature Beans \\
\hline 88 & 74 & 89 & 89 & Mature Beans \\
\hline 89 & 113 & 123 & 134 & Mature Beans \\
\hline 90 & 137 & 154 & 157 & Mature Beans \\
\hline 91 & 109 & 121 & 131 & Mature Beans \\
\hline 92 & 125 & 140 & 145 & Mature Beans \\
\hline 93 & 110 & 121 & 129 & Mature Beans \\
\hline 94 & 109 & 120 & 131 & Mature Beans \\
\hline 95 & 111 & 122 & 130 & Mature Beans \\
\hline 96 & 110 & 120 & 131 & Mature Beans \\
\hline 97 & 108 & 121 & 125 & Mature Beans \\
\hline 98 & 98 & 107 & 116 & Mature Beans \\
\hline 99 & 143 & 163 & 159 & Mature Beans \\
\hline 100 & 123 & 138 & 142 & Mature Beans \\
\hline 1 & 113 & 123 & 134 & Immature Beans \\
\hline 2 & 137 & 154 & 157 & Immature Beans \\
\hline 3 & 109 & 121 & 131 & Immature Beans \\
\hline 4 & 125 & 140 & 145 & Immature Beans \\
\hline 5 & 110 & 121 & 129 & Immature Beans \\
\hline 6 & 109 & 120 & 131 & Immature Beans \\
\hline 7 & 111 & 122 & 130 & Immature Beans \\
\hline 8 & 110 & 120 & 131 & Immature Beans \\
\hline 9 & 108 & 121 & 125 & Immature Beans \\
\hline 10 & 98 & 107 & 116 & Immature Beans \\
\hline 11 & 143 & 163 & 159 & Immature Beans \\
\hline 12 & 123 & 138 & 142 & Immature Beans \\
\hline 13 & 136 & 155 & 153 & Immature Beans \\
\hline
\end{tabular}


William R. Eustaquio et al., International Journal of Emerging Trends in Engineering Research, 8(7), July 2020, 3016 - 3022

\begin{tabular}{|c|c|c|c|c|}
\hline 14 & 119 & 133 & 139 & Immature Beans \\
\hline 15 & 130 & 147 & 146 & Immature Beans \\
\hline 16 & 100 & 108 & 118 & Immature Beans \\
\hline 17 & 123 & 140 & 140 & Immature Beans \\
\hline 18 & 113 & 123 & 134 & Immature Beans \\
\hline 19 & 111 & 124 & 126 & Immature Beans \\
\hline 20 & 113 & 125 & 130 & Immature Beans \\
\hline 21 & 140 & 158 & 154 & Immature Beans \\
\hline 22 & 124 & 138 & 138 & Immature Beans \\
\hline 23 & 116 & 129 & 132 & Immature Beans \\
\hline 24 & 127 & 144 & 140 & Immature Beans \\
\hline 25 & 121 & 136 & 134 & Immature Beans \\
\hline 26 & 96 & 101 & 115 & Immature Beans \\
\hline 27 & 120 & 132 & 138 & Immature Beans \\
\hline 28 & 150 & 168 & 164 & Immature Beans \\
\hline 29 & 119 & 130 & 138 & Immature Beans \\
\hline 30 & 147 & 165 & 161 & Immature Beans \\
\hline 31 & 124 & 139 & 139 & Immature Beans \\
\hline 32 & 129 & 144 & 144 & Immature Beans \\
\hline 33 & 113 & 126 & 132 & Immature Beans \\
\hline 34 & 95 & 101 & 113 & Immature Beans \\
\hline 35 & 98 & 106 & 116 & Immature Beans \\
\hline 36 & 119 & 131 & 136 & Immature Beans \\
\hline 37 & 145 & 164 & 156 & Immature Beans \\
\hline 38 & 113 & 125 & 129 & Immature Beans \\
\hline 39 & 122 & 136 & 136 & Immature Beans \\
\hline 40 & 130 & 149 & 142 & Immature Beans \\
\hline 41 & 149 & 167 & 161 & Immature Beans \\
\hline 42 & 113 & 125 & 130 & Immature Beans \\
\hline 43 & 107 & 117 & 127 & Immature Beans \\
\hline 44 & 104 & 116 & 117 & Immature Beans \\
\hline 45 & 95 & 105 & 111 & Immature Beans \\
\hline 46 & 121 & 138 & 135 & Immature Beans \\
\hline 47 & 133 & 150 & 148 & Immature Beans \\
\hline 48 & 122 & 136 & 139 & Immature Beans \\
\hline 49 & 105 & 116 & 120 & Immature Beans \\
\hline 50 & 126 & 143 & 141 & Immature Beans \\
\hline 51 & 106 & 120 & 127 & Immature Beans \\
\hline 52 & 108 & 119 & 129 & Immature Beans \\
\hline 53 & 128 & 149 & 150 & Immature Beans \\
\hline 54 & 106 & 120 & 127 & Immature Beans \\
\hline 55 & 126 & 145 & 148 & Immature Beans \\
\hline 56 & 114 & 133 & 134 & Immature Beans \\
\hline 57 & 98 & 109 & 118 & Immature Beans \\
\hline
\end{tabular}

\begin{tabular}{|c|c|c|c|c|}
\hline 58 & 133 & 155 & 152 & Immature Beans \\
\hline 59 & 138 & 160 & 155 & Immature Beans \\
\hline 60 & 130 & 151 & 150 & Immature Beans \\
\hline 61 & 124 & 142 & 146 & Immature Beans \\
\hline 62 & 115 & 132 & 136 & Immature Beans \\
\hline 63 & 110 & 125 & 130 & Immature Beans \\
\hline 64 & 110 & 124 & 130 & Immature Beans \\
\hline 65 & 99 & 108 & 119 & Immature Beans \\
\hline 66 & 143 & 164 & 162 & Immature Beans \\
\hline 67 & 132 & 153 & 148 & Immature Beans \\
\hline 68 & 138 & 163 & 152 & Immature Beans \\
\hline 69 & 123 & 141 & 142 & Immature Beans \\
\hline 70 & 127 & 149 & 144 & Immature Beans \\
\hline 71 & 109 & 126 & 126 & Immature Beans \\
\hline 72 & 97 & 107 & 118 & Immature Beans \\
\hline 73 & 123 & 143 & 142 & Immature Beans \\
\hline 74 & 141 & 161 & 157 & Immature Beans \\
\hline 75 & 124 & 143 & 140 & Immature Beans \\
\hline 76 & 138 & 157 & 156 & Immature Beans \\
\hline 77 & 135 & 159 & 147 & Immature Beans \\
\hline 78 & 120 & 136 & 139 & Immature Beans \\
\hline 79 & 101 & 113 & 119 & Immature Beans \\
\hline 80 & 138 & 162 & 153 & Immature Beans \\
\hline 81 & 115 & 132 & 134 & Immature Beans \\
\hline 82 & 121 & 139 & 140 & Immature Beans \\
\hline 83 & 124 & 143 & 143 & Immature Beans \\
\hline 84 & 116 & 133 & 134 & Immature Beans \\
\hline 85 & 99 & 108 & 118 & Immature Beans \\
\hline 86 & 121 & 139 & 141 & Immature Beans \\
\hline 87 & 130 & 149 & 144 & Immature Beans \\
\hline 88 & 147 & 168 & 162 & Immature Beans \\
\hline 89 & 133 & 154 & 146 & Immature Beans \\
\hline 90 & 119 & 136 & 138 & Immature Beans \\
\hline 91 & 124 & 145 & 140 & Immature Beans \\
\hline 92 & 129 & 152 & 143 & Immature Beans \\
\hline 93 & 112 & 129 & 131 & Immature Beans \\
\hline 94 & 93 & 102 & 114 & Immature Beans \\
\hline 95 & 116 & 134 & 133 & Immature Beans \\
\hline 96 & 116 & 131 & 134 & Immature Beans \\
\hline 97 & 109 & 128 & 125 & Immature Beans \\
\hline 98 & 127 & 147 & 146 & Immature Beans \\
\hline 99 & 90 & 100 & 104 & Immature Beans \\
\hline 100 & 110 & 126 & 129 & Immature Beans \\
\hline
\end{tabular}

